\title{
Aplicação da Metodologia do Grau de Deterioração da Estrutura (GDE/UnB) em um viaduto: estudo de caso no município de Santa Maria/RS
}

\author{
C. de $\operatorname{Vargas}^{1 *}$, G. D. Brackmann ${ }^{1}$, G. F. dos $\operatorname{Santos}^{1}$, E. M. de Carli ${ }^{1}$, A. S. de $\operatorname{Vargas}^{2}$, R. C. A. \\ de Lima ${ }^{2}$ \\ *Autor de Contato: camile.vargas@ufsm.br
}

\footnotetext{
${ }^{1}$ Estudante de Mestrado, Programa de Pós-Graduação em Engenharia Civil, Universidade Federal de Santa Maria, Santa Maria, Brasil

${ }^{2}$ Professor, Programa de Pós-Graduação em Engenharia Civil, Universidade Federal de Santa Maria, Santa Maria, Brasil
}

\begin{abstract}
RESUMO
Viadutos são estruturas sujeitas a ações externas, como o intemperismo ambiental, resultando em danos que podem diminuir sua vida útil consideravelmente caso não ocorram manutenções periódicas para sua conservação. Nesse sentido, o presente trabalho teve como objetivo analisar e identificar as manifestações patológicas de um viaduto localizado no município de Santa Maria (RS), o qual faz a ligação entre dois bairros importantes da cidade, utilizando a metodologia GDE/UnB, na versão proposta por Verly (2015). A aplicação da metodologia GDE/UnB possibilitou a obtenção de resultados condizentes com os dados obtidos na vistoria in loco, permitindo a determinação de quais seriam as ações imediatas que devem ser priorizadas visando reestabelecer a conservação e o desempenho da estrutura objeto deste estudo.
\end{abstract}

Palavras-chave: viaduto; manifestação patológica; grau de deterioração; manutenção. 


\begin{abstract}
Viaducts are structures subject to external actions, such as environmental weathering, resulting in damage that can considerably shorten its useful life if there are no periodic maintenance for its conservation. In this sense, this study aimed to analyze and identify the pathological manifestations of a viaduct located in Santa Maria city (RS), which connects two important neighborhoods of the city, using the GDE/UnB methodology, in the version proposed by Verly (2015). The application of the GDE/UnB methodology made it possible to obtain results consistent with the data obtained in the on-site survey, allowing the determination of what would be the immediate actions that should be prioritized in order to re-establish the conservation and performance of the analyzed structure.
\end{abstract}

Keywords: viaduct; pathological manifestation; degree of deterioration; maintenance.

\title{
RESUMEN
}

Los viaductos son estructuras sometidas a acciones externas, como la intemperie ambiental, resultando en daños que pueden acortar considerablemente su vida útil si no se realizan mantenimientos periódicos para su conservación. En este sentido, este estudio objetivas analizar e identificar las manifestaciones patológicas de un viaducto ubicado en la ciudad de Santa María (RS), que conecta dos importantes barrios de la ciudad, utilizando la metodología GDE/UnB, en la versión propuesta por Verly. (2015). La aplicación de la metodología GDE/UnB permitió obtener resultados consistentes con los datos obtenidos en el relevamiento in situ, permitiendo determinar cuáles serían las acciones inmediatas que se deben priorizar para restablecer la conservación y el desempeño de la estructura analizada.

Palabras clave: viaducto; manifestación patológica; grado de deterioro; mantenimiento.

\section{INTRODUÇÃO}

Viadutos são Obras de Arte Especiais (OAE) de grande importância dentro da estrutura viária de uma cidade, uma vez que viabilizam o deslocamento sobre obstáculos e assim facilitam a logística de tráfego encurtando distância e realizando a interligação entre localidades. Na maioria dos casos, a manutenção dessas estruturas é negligenciada e à medida que a estrutura se deteriora naturalmente, a falta de manutenção acelera o surgimento de patologias, denotando a importância de intervenções periódicas.

Entre os vários métodos disponíveis para a avaliação da degradação de estruturas, existe a metodologia do Grau de Deterioração da Estrutura (GDE/UnB), que classifica os danos e avalia a deterioração de estruturas de concreto. Essa avaliação é feita a partir da inspeção da estrutura, com a atribuição de notas conforme a intensidade dos danos observados nos elementos estruturais. Assim, o objetivo deste trabalho é aplicar a metodologia GDE/UnB à estrutura de um viaduto situado na cidade de Santa Maria/RS, por meio de uma inspeção das condições gerais da estrutura. O viaduto alvo da análise é responsável pela ligação de dois bairros da cidade, sendo, portanto, importante realizar o acompanhamento de seu estado de conservação regularmente, para que sejam realizadas as devidas manutenções quando necessário. 


\section{METODOLOGIA GDE/UnB}

A metodologia a ser aplicada no presente artigo é a do Grau de Deterioração da Estrutura (GDE/UnB), desenvolvida inicialmente por Castro (1994). A evolução da metodologia GDE/UnB ocorreu com Lopes (1998) e Fonseca (2007), que utilizaram a mesma para avaliar estruturas de edificações, assim como com Euqueres (2011) e Verly (2015), que apresentaram excelentes resultados de aplicação da metodologia em OAE. O fluxograma apresentado na Figura 1 mostra a sequência de aplicação da metodologia GDE/UnB, assim como os respectivos fatores e equações que serão melhor detalhados nesta seção.

\subsection{Fator de ponderação de dano $\left(F_{p}\right)$}

A metodologia GDE/UnB propõe a verificação da ocorrência ou não de determinados tipos de danos nos elementos, sendo eles a carbonatação, o cobrimento deficiente, a contaminação por cloretos, a corrosão de armaduras, a desagregação, o deslocamento por empuxo, o desplacamento, os desvios de geometria, a eflorescência, a falha de concretagem, as fissuras, as flechas excessivas, a impermeabilização deficiente, a infiltração de água, as manchas, a obstrução de juntas de dilatação, os recalques e os sinais de esmagamento do concreto. Os valores de $\mathrm{F}_{\mathrm{p}}$ podem variar de 1 a 5 , sendo definidos antes da inspeção com exceção do dano relativo às fissuras, em que o valor de $\mathrm{F}_{\mathrm{p}}$ é definido no momento da inspeção de acordo com a natureza das fissuras identificadas nos elementos. Os elementos estruturais são divididos em famílias típicas para fins de análise e para cada uma delas, a metodologia GDE/UnB fornece os fatores de ponderação do dano $\left(\mathrm{F}_{\mathrm{p}}\right)$ relativos a cada tipo de dano que pode ser identificado nos elementos, o quais têm como finalidade quantificar uma importância relativa de um determinado dano nas condições gerais de segurança, funcionalidade e estética dos elementos de uma família (Castro, 1994).

\subsection{Fator de intensidade do dano $\left(F_{i}\right)$}

$\mathrm{Na}$ atribuição desse fator é importante observar o ambiente onde se encontra o dano, a profundidade do mesmo, assim como as deformações aparentes, de modo a diminuir a interferência da subjetividade da análise que é feita visualmente no momento da vistoria. $\mathrm{O}$ fator de intensidade do dano tem como objetivo classificar a gravidade e a evolução isolada do dano em um determinado elemento, variando de 0 a 4 (zero a quatro), conforme Tabela 1, sendo que 0 (zero) é o valor atribuído ao elemento que não possui nenhuma lesão de um determinado tipo de dano (corrosão de armaduras, cobrimento insuficiente, etc.), enquanto que 4 (quatro), o valor máximo, é atribuído ao elemento que apresentar uma determinada lesão em estado crítico.

Tabela 1. Classificação do fator de intensidade do dano.

\begin{tabular}{|c|c|}
\hline Estado do elemento & $\mathbf{F}_{\mathbf{i}}$ \\
\hline Elemento sem lesões & 0 \\
\hline Elemento com lesões leves & 1 \\
\hline Elemento com lesões toleráveis & 2 \\
\hline Elementos com lesões graves & 3 \\
\hline Elemento em estado crítico & 4 \\
\hline
\end{tabular}

Fonte: Verly (2015). 


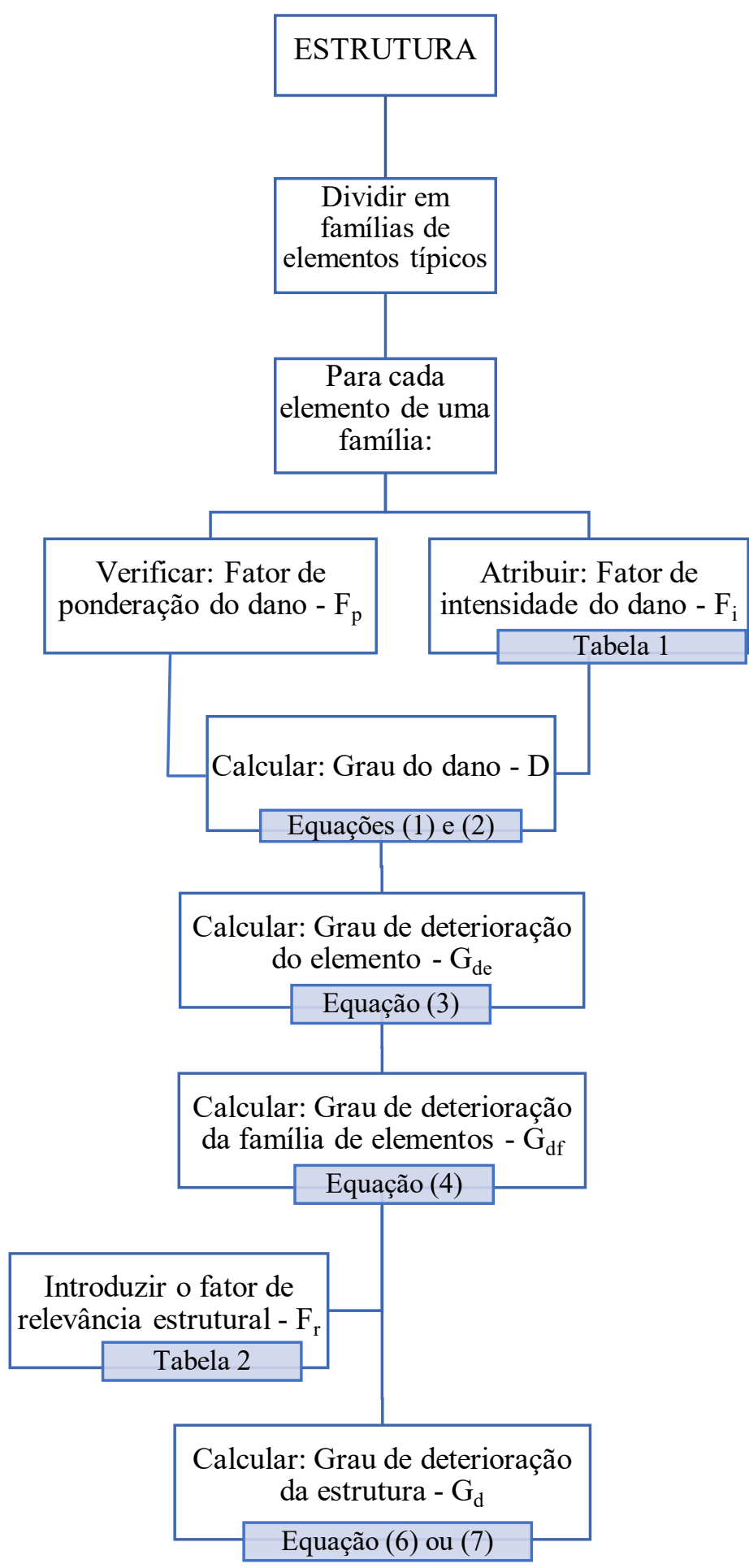

Figura 1. Fluxograma de aplicação da metodologia GDE/UnB (adaptado de Castro, 1994).

\subsection{Grau de dano (D)}

O grau de dano (D) foi desenvolvido com a finalidade de quantificar o processo de deterioração da estrutura. É o fator que relaciona o Fator de Intensidade $\left(\mathrm{F}_{\mathrm{i}}\right)$ e o Fator de Ponderação $\left(\mathrm{F}_{\mathrm{p}}\right)$ a partir das Equações (1) e (2). 


$$
\begin{array}{lll}
D=0,8 \cdot F_{i} \cdot F_{p} & \text { para } & F_{i} \leq 2,0 \\
D=\left(12 \cdot F_{i}-28\right) \cdot F_{p} & \text { para } & F_{i}>2,0
\end{array}
$$

\subsection{Grau de deterioração do elemento $\left(G_{d e}\right)$}

O grau de deterioração do elemento $\left(\mathrm{G}_{\mathrm{de}}\right)$ é o parâmetro que indica o nível de degradação de cada elemento individualmente. Deve ser feita a análise do elemento estrutural sob o efeito de todos os danos nele existentes, utilizando a Equação (3) que foi proposta por Lopes (1998).

$$
G_{d e}=D_{m a ́ x} \cdot\left[1+\frac{\left(\sum_{i=1}^{n} D_{i}\right)-D_{m a ́ x}}{\sum_{i=1}^{n} D_{i}}\right]
$$

Onde: $\mathrm{D}_{\mathrm{i}}=$ grau de dano de ordem $\mathrm{i} ; \mathrm{D}_{\text {máx }}=$ maior dano identificado no elemento; $\mathrm{n}=$ número de danos encontrados em um elemento.

\subsection{Grau de deterioração de uma família de elementos $\left(G_{d f}\right)$}

O grau de deterioração de uma família de elementos $\left(\mathrm{G}_{\mathrm{df}}\right)$ representa a deterioração de um conjunto de elementos pertencentes a uma mesma família. Para seu cálculo são considerados os valores de $\mathrm{G}_{\text {de }}$ de cada elemento da família que está sendo analisada, conforme a Equação (4).

$$
G_{d f}=G_{d e, m a ́ x} \cdot\left[1+\frac{\left(\sum_{i=1}^{m} G_{d e, i}\right)-G_{d e, m a ́ x}}{\sum_{i=1}^{m} G_{d e, i}}\right]
$$

Onde: $\mathrm{G}_{\mathrm{de}, \text { máx }}=$ grau de deterioração máxima do elemento; $\mathrm{G}_{\mathrm{de}, \mathrm{i}}=$ grau de deterioração do elemento de ordem $\mathrm{i} ; \mathrm{m}=$ número de elementos da família.

\subsection{Fator de relevância estrutural $\left(F_{r}\right)$}

O fator de relevância estrutural tem como papel considerar a influência de cada família em função de sua importância na estabilidade estrutural e bom desempenho da estrutura. Dessa forma, o valor do $F_{r}$ depende do sistema estrutural em análise, sendo recomendados por Verly (2015) os valores da Tabela 2 .

Tabela 2. Fatores de relevância estrutural.

\begin{tabular}{|l|c|}
\hline \multicolumn{1}{|c|}{ Tipo de elemento } & Fr $_{\mathbf{r}}$ \\
\hline Barreiras de defensas, guarda-corpo, guarda-rodas, pista de rolamento & 1,0 \\
\hline Juntas de dilatação & 2,0 \\
\hline Transversinas, cortinas, alas & 3,0 \\
\hline Lajes, fundações, vigas secundárias, aparelhos de apoio & 4,0 \\
\hline Vigas principais e pilares & 5,0 \\
\hline
\end{tabular}

Fonte: Verly (2015).

\subsection{Grau de Deterioração da Estrutura $\left(G_{d}\right)$}

Para obter o grau de deterioração da estrutura $\left(\mathrm{G}_{\mathrm{d}}\right)$, deve ser considerado o fator de relevância de cada família e o grau de deterioração da família. A Equação (5) fornece o grau de deterioração da família majorado $(K)$, dado pelo produto do $G_{d, f}$ e $F_{r}$. Enquanto Fonseca (2007) propôs a Equação 
(6) para determinar o grau de deterioração da estrutura $\left(G_{d}\right)$, em que os valores de $G_{d f}$ são ponderados pelos respectivos fatores de relevância estrutural desprezando-se os valores de $G_{d e}$ menores que 15 no cálculo do $\mathrm{G}_{\mathrm{df}}$, Verly (2015) modificou tal equação e propôs a Equação (7) para o cálculo do grau de deterioração da estrutura modificado $\left(\mathrm{G}_{\mathrm{d}, \bmod }{ }^{*}\right)$, e definiu que todos os valores de $G_{\text {de }}$ devem ser considerados no cálculo do $G_{d f}$.

$$
\begin{aligned}
& K_{i}=G_{d f, i} \cdot F_{r, i} \\
& G_{d}=\frac{\sum_{i=1}^{k} K_{i}}{\sum_{i=1}^{k} F_{r, i}} \\
& G_{d, \bmod }{ }^{*}=\frac{K_{\text {máx }}}{7,07} \cdot \sqrt{1+\frac{\left(\sum_{i=1}^{k} K_{i}\right)-K_{m a ́ x}}{\sum_{i=1}^{k} K_{i}}}
\end{aligned}
$$

Após o cálculo do $\mathrm{G}_{\mathrm{d}}\left(\right.$ ou $\left.\mathrm{G}_{\mathrm{d} \text {,mod }}{ }^{*}\right)$, a estrutura pode ser classificada em níveis conforme a Tabela 3,

\begin{tabular}{|c|c|c|}
\hline $\begin{array}{c}\text { Nível de } \\
\text { deterioração }\end{array}$ & $\mathbf{G}_{\mathbf{d}}$ & Ações recomendadas \\
\hline Baixo & $0-15$ & $\begin{array}{l}\text { Estado aceitável. } \\
\text { Manutenção preventiva. }\end{array}$ \\
\hline Médio & $15-50$ & $\begin{array}{c}\text { Definir prazo e natureza de nova inspeção. } \\
\text { Planejar intervenção em longo prazo (máx. } 2 \text { anos). }\end{array}$ \\
\hline Alto & $50-80$ & $\begin{array}{l}\text { Definir prazo para inspeção especializada. } \\
\text { Planejar intervenção em médio prazo (máx. } 1 \text { ano). }\end{array}$ \\
\hline Sofrível & $80-100$ & $\begin{array}{l}\text { Definir prazo para inspeção especializada rigorosa. } \\
\text { Planejar intervenção em curto prazo (máx. } 6 \text { meses). }\end{array}$ \\
\hline Crítico & $\geq 100$ & $\begin{array}{l}\text { Inspeção especializada imediata e medidas emergenciais (alívio } \\
\text { de cargas, escoramento, etc.). Planejar intervenção imediata. }\end{array}$ \\
\hline
\end{tabular}
onde constam recomendações de ações a serem tomadas em cada caso.

Tabela 3. Classificação dos níveis de deterioração da estrutura e ações recomendadas.

Fonte: Verly (2015).

\section{CARACTERIZAÇÃO DO OBJETO DE ESTUDO}

A OAE analisada neste estudo de caso está localizada na Avenida Assis Brasil, sobre os trilhos da ferrovia que passa pelo município, entre os bairros Centro e Itararé, fazendo a interligação dos mesmos, na cidade de Santa Maria, no estado do Rio Grande do Sul, Brasil. A Figura 2 apresenta a localização da estrutura no bairro Itararé.

A estrutura do viaduto é composta de vigas, longarinas, transversinas e lajes em concreto armado, moldado in loco. Em relação à seção transversal, essa possui uma largura total de 9,70m, largura de pista de 7,30m, passeios de 1,20 $\mathrm{m}$ de comprimento e $0,18 \mathrm{~m}$ de altura além de guardas - corpo de $1,44 \mathrm{~m}$ cada, dispostos lado a lado, com $0,90 \mathrm{~m}$ de altura, conforme detalhado na Figura 3 . $\mathrm{O}$ tipo de fundação do viaduto e as condições atuais dessa estrutura não puderam ser verificadas. 


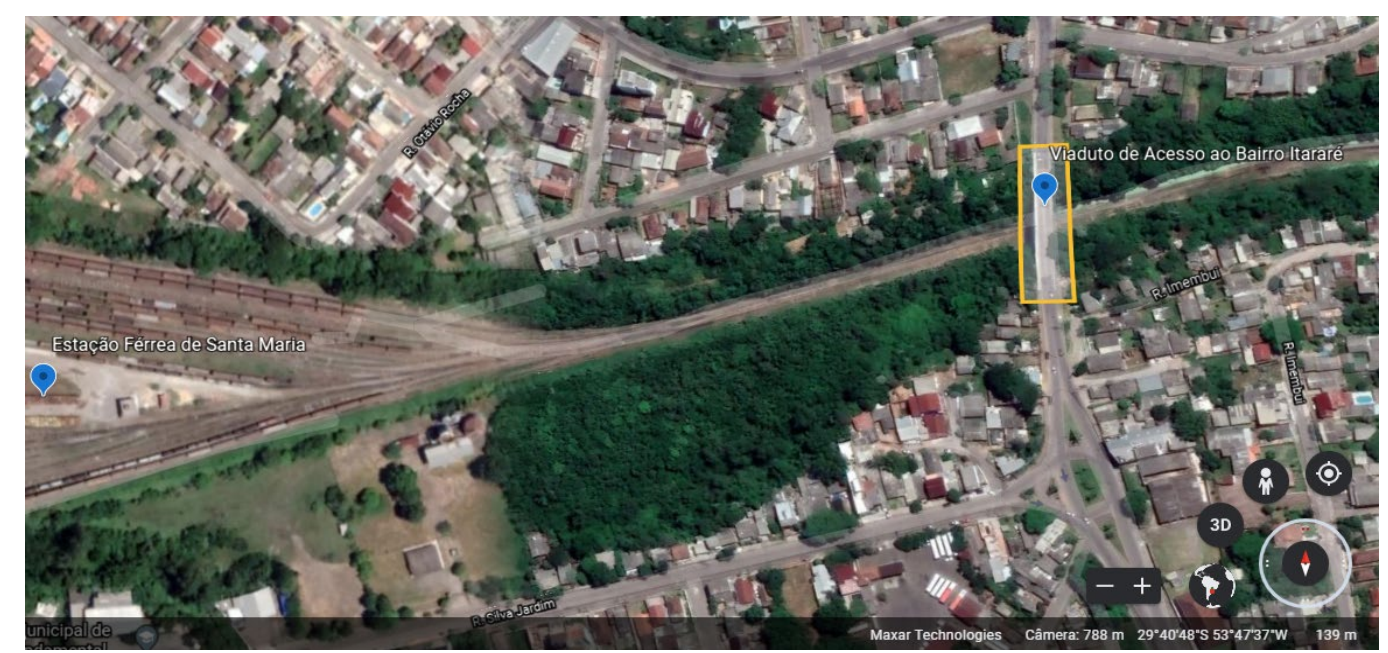

Figura 2. Localização da OAE em imagem de satélite. Fonte: Google Earth (2021).

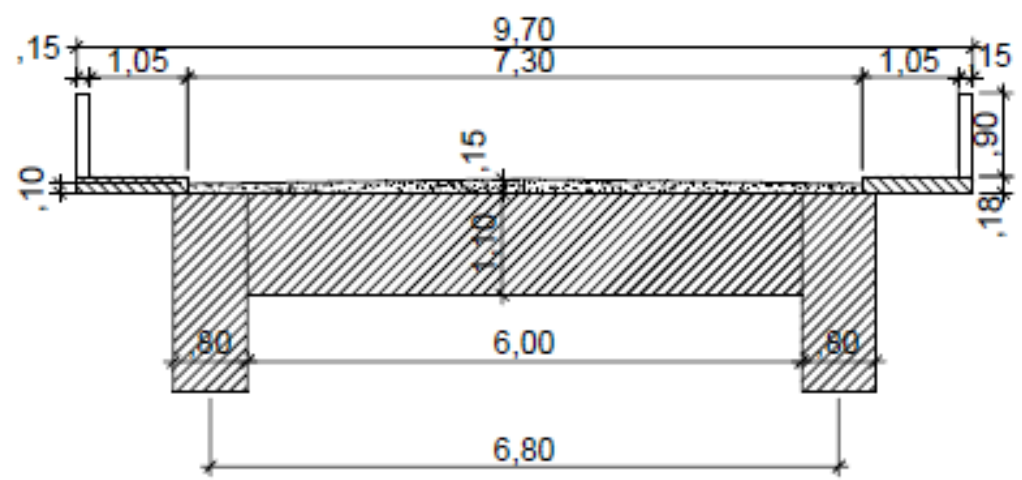

Figura 3. Seção Transversal do Viaduto do Itararé localizado na cidade de Santa Maria/RS.

\subsection{Mesoestrutura}

A mesoestrutura do viaduto analisado é composta por cinco estruturas aporticadas, cada uma composta por dois pilares $(80 \times 50 \mathrm{~cm})$ e uma viga $(50 \times 110 \mathrm{~cm})$, todos de seção constante e em concreto armado (Figura 4).

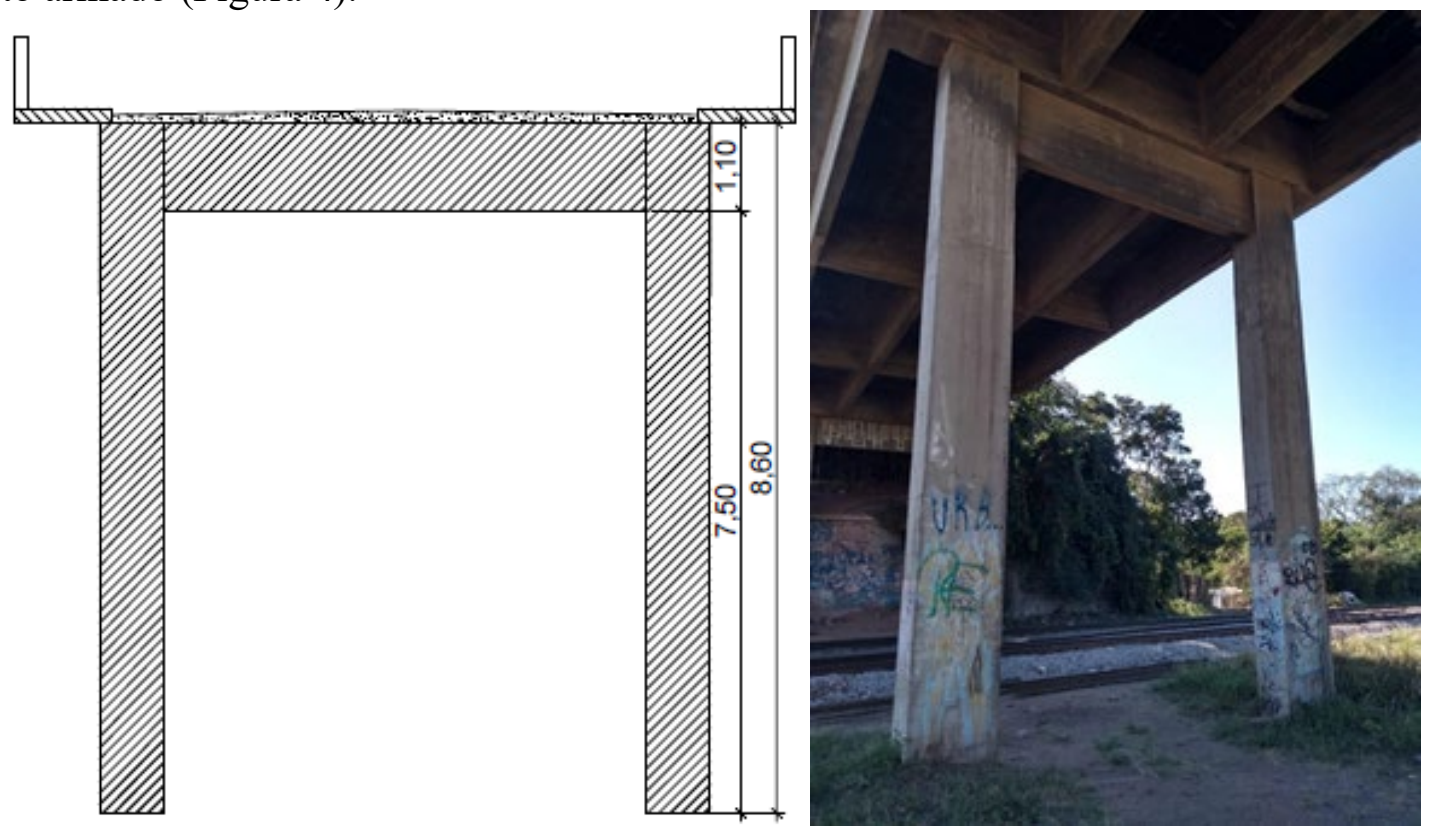

Figura 4. Pórticos que compõem o viaduto. 


\subsection{Superestrutura}

A superestrutura do viaduto, objeto analisado neste artigo, é composta de um tabuleiro de lajes maciças de concreto armado, travessas, longarinas e transversinas (Figura 5).

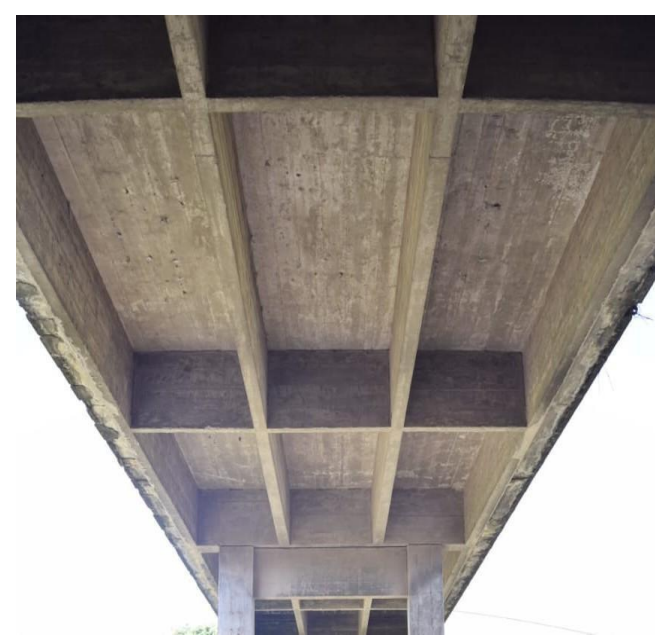

(a)

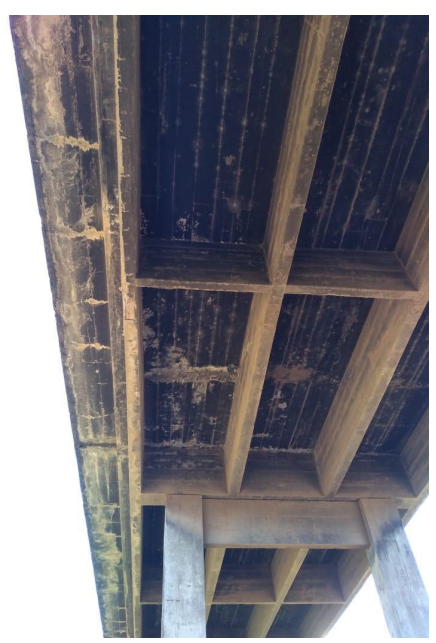

(b)

Figura 5. Tabuleiro de lajes maciças de concreto armado, travessas, longarinas e transversinas que compõem a estrutura do viaduto.

\section{INSPEÇÃO DA OAE}

A inspeção foi realizada de forma visual, para caracterizar a geometria dos elementos e identificar fenômenos patológicos existentes. Para fins de análise, a OAE foi dividida nas seguintes famílias: pilares, travessas, longarinas, transversinas, lajes, pista de rolamento, guarda-roda e guarda-corpo.

\subsection{Pilares}

Em geral, os pilares apresentavam pontos de desplacamento e manchas evidentes. Na base do Pilar 5 (P5), conforme mostra a Figura 6, foram identificados pontos de exposição da armadura, tanto da principal como dos estribos e presença de corrosão. Seguindo a metodologia GDE/UnB, para o desplacamento, o fator de intensidade adotado foi igual a 3 por serem lascamentos de grandes proporções, com exposição da armadura. Para a corrosão, adotou-se o fator de intensidade igual a 2, uma vez que foram identificadas apenas manifestações leves, com pequenas manchas.

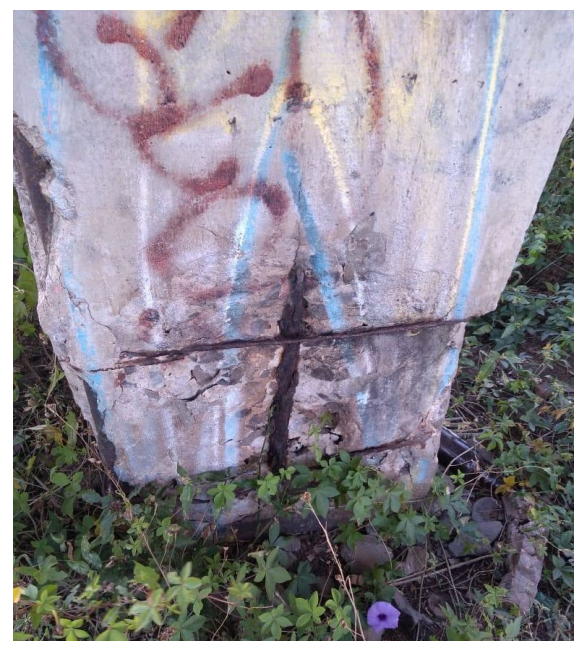

Figura 6. Pilar 5 com armaduras expostas. 


\subsection{Travessas, Longarinas e Transversinas}

As travessas, longarinas e transversinas apresentavam pontos de corrosão das armaduras, desagregação, desplacamento, infiltração de água e manchas, sendo que alguns pontos dessas manifestações patológicas são mais acentuados do que outros. As longarinas localizadas nas bordas (L1 e L4) possuíam manchas mais significativas devido a falhas de drenagem. As travessas apresentavam situações semelhantes em geral, no entanto, algumas delas possuíam pontos de corrosão de armadura que não foram identificados em outras. Em relação às transversinas, a T4 encontrava-se em situação mais crítica quando comparada com todas as outras, apresentando desde infiltração de água e manchas até desagregação, desplacamento e corrosão de armaduras.

\subsection{Lajes}

Para fins de análise dividiu-se a estrutura que compõe a laje do viaduto em quatro vãos. Observouse que os vãos intermediários possuíam manchas mais intensas devido à umidade, adotando-se $\mathrm{F}_{\mathrm{i}}$ igual a 4 para estas e $F_{i}$ igual a 3 para as manchas visualizadas nos vãos das extremidades. Devido à considerável infiltração de água, resultando em grandes manchas, o $\mathrm{F}_{\mathrm{p}}$ utilizado no cálculo para essa manifestação patológica foi 3. A Figura 7(a) mostra uma fotografia do tabuleiro em 2018 e a Figura 7(b) em 2021, comparação que permite identificar o avanço rápido das manifestações patológicas ao longo dos últimos dois anos devido, principalmente, à ausência de manutenção periódica.

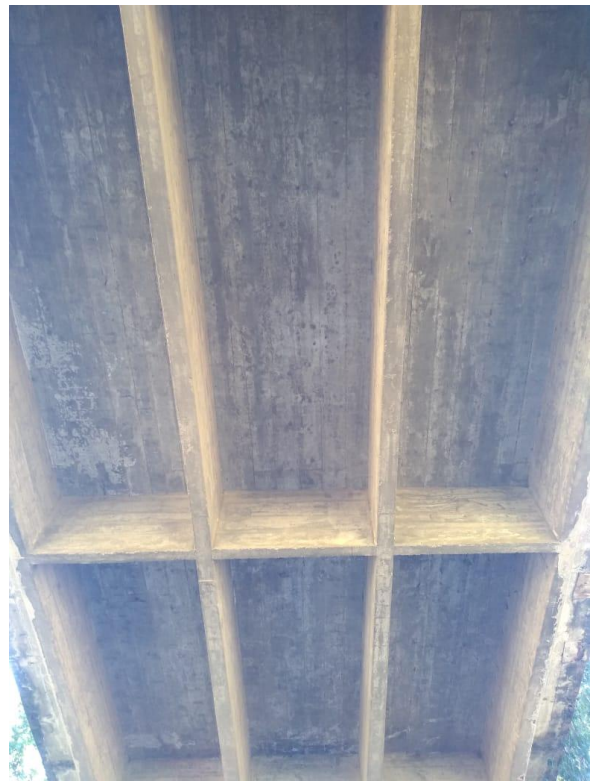

(a)

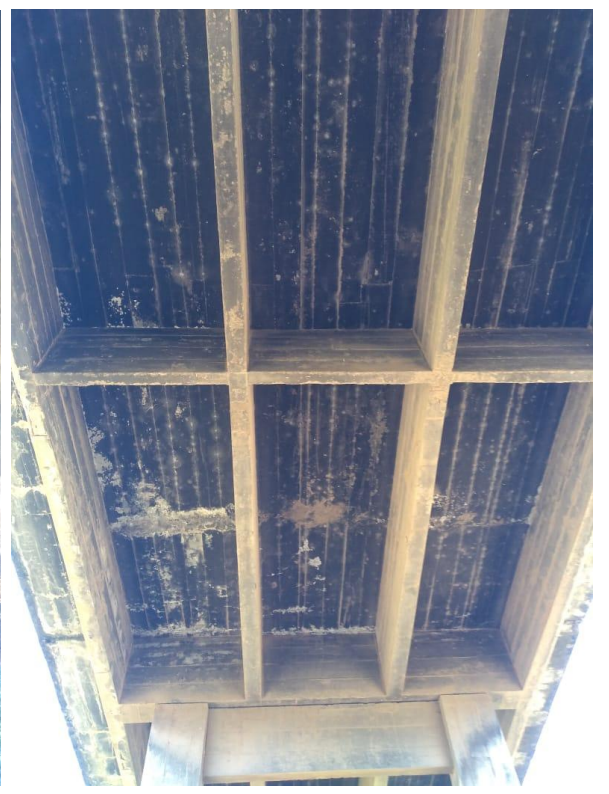

(b)

Figura 7. Comparação do Tabuleiro do Viaduto do Itararé em 2018 (a) e 2021 (b).

\subsection{Pista de rolamento e Juntas de dilatação}

A pista de rolamento apresenta desgaste superficial em estado avançado, conforme a Figura 8(a), sendo atribuído um fator de intensidade igual a 3 seguindo as recomendações da metodologia GDE/UnB. Além disso, foram verificadas descontinuidades na pista.

Em relação às juntas de dilatação, como é possível observar na Figura 8(b), essas possuem obstruções com perda de elasticidade do material da junta, acompanhada do início de fissuras paralelas às juntas nas lajes e paredes adjacentes, o que justifica a atribuição do fator de intensidade 2 para esse dano. 


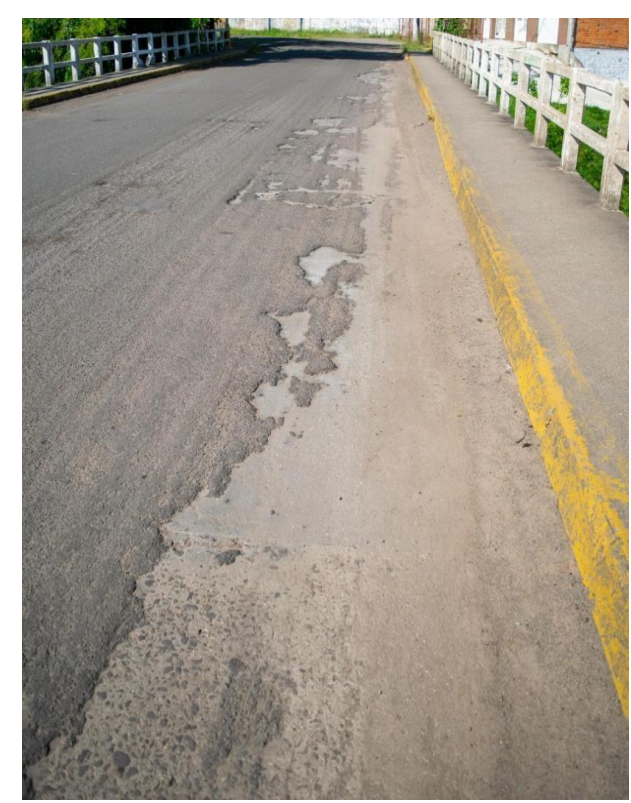

(a)

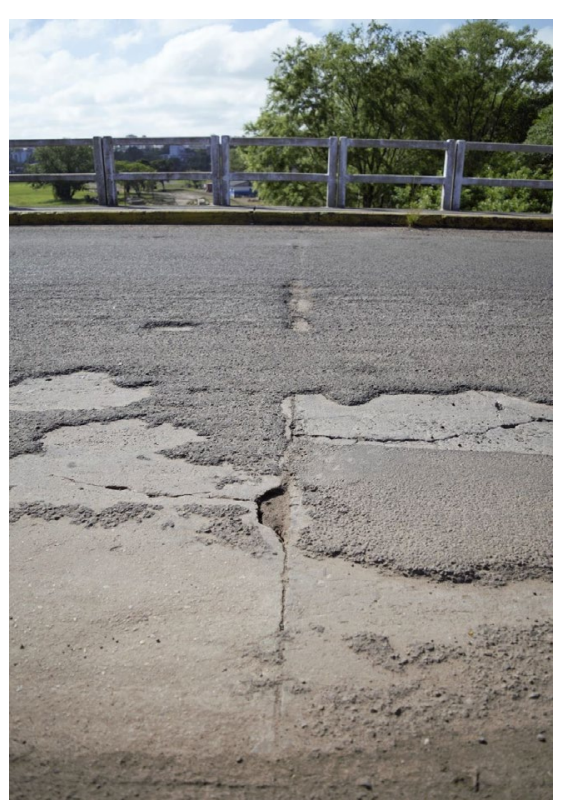

(b)

Figura 8. Pista de rolamento (a) e junta de dilatação (b)

\subsection{Guarda-rodas e guarda-corpo}

Os guarda-rodas apresentavam bom estado de conservação, sendo identificadas apenas algumas manchas e pequenos pontos de desagregação não intensos. No entanto, nos guarda-corpos, foram identificados, em vários pontos, cobrimento insuficiente, em que era possível visualizar os agregados graúdos utilizados no concreto que compõe o elemento (Figura 9). Em outros pontos, a deterioração era ainda mais intensa, com desplacamentos caracterizados por apresentar lascamentos acentuados com perda relevante da seção, tendo sido atribuído, portanto, $\mathrm{F}_{\mathrm{i}}$ igual a 4 para esse dano, assim como para a corrosão que já havia levado à uma perda relevante de seção da armadura.
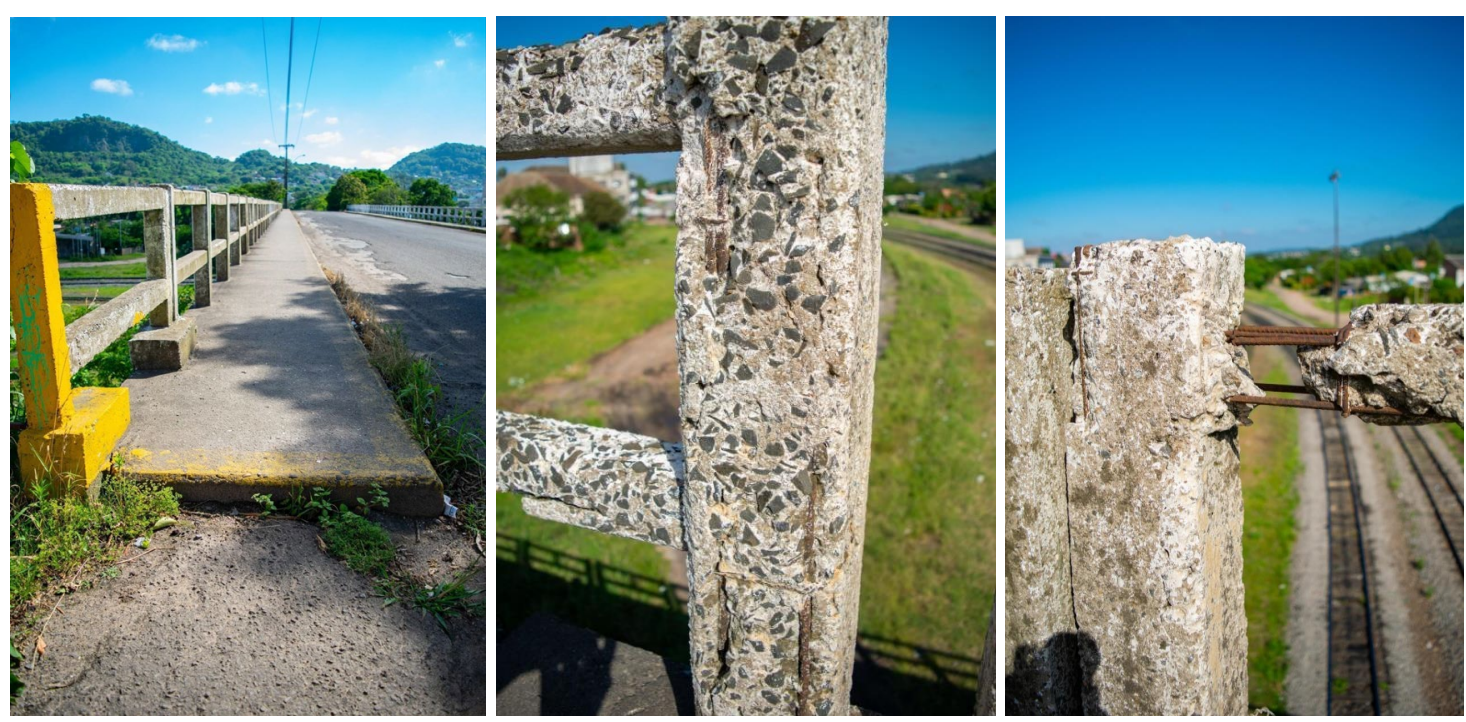

Figura 9. Detalhe dos danos no guarda-corpo. 


\section{RESULTADOS E DISCUSSÃO}

Na Tabela 4 são apresentados os valores do Fator de Relevância $\left(F_{r}\right)$ e Fator de Ponderação do Dano $\left(F_{p}\right)$ utilizados, seguindo a metodologia GDE/UnB, assim como os Fatores de Intensidade do Dano $\left(\mathrm{F}_{\mathrm{i}}\right)$ adotados para as manifestações patológicas verificadas em cada elemento e respectivos resultados do cálculo do Grau de Dano (D) e Grau de Deterioração do Elemento $\left(\mathrm{G}_{\mathrm{de}}\right)$.

Tabela 4. Aplicação da metodologia GDE/UnB no viaduto do Itararé.

\begin{tabular}{|c|c|c|c|c|c|c|c|}
\hline Família & $\mathbf{F}_{\mathbf{r}}$ & Elementos & Manifestação Patológica & $\mathbf{F}_{\mathbf{p}}$ & $\mathbf{F}_{\mathbf{i}}$ & $\mathbf{D}$ & $\mathbf{G}_{\mathbf{d e}}$ \\
\hline \multirow{10}{*}{ 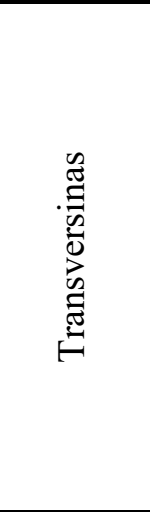 } & \multirow{10}{*}{3} & \multirow{2}{*}{$\mathrm{T} 1 ; \mathrm{T} 13$} & Infiltração de água & 3 & 1 & 3 & \multirow{2}{*}{8,00} \\
\hline & & & Manchas & 3 & 2 & 6 & \\
\hline & & \multirow{3}{*}{$\begin{array}{c}\text { T2;T3;T5;T6; } \\
\text { T7;T8;T9; } \\
\text { T10;T11;T12 }\end{array}$} & Corrosão de armaduras & 5 & 2 & 10 & \multirow{3}{*}{14,74} \\
\hline & & & Infiltração de água & 3 & 1 & 3 & \\
\hline & & & Manchas & 3 & 2 & 6 & \\
\hline & & \multirow{5}{*}{$\mathrm{T} 4$} & Corrosão de armaduras & 5 & 2 & 10 & \multirow{5}{*}{36,24} \\
\hline & & & Desagregação & 3 & 2 & 6 & \\
\hline & & & Desplacamento & 3 & 3 & 24 & \\
\hline & & & Infiltração de água & 3 & 1 & 3 & \\
\hline & & & Manchas & 3 & 2 & 6 & \\
\hline \multirow{6}{*}{ 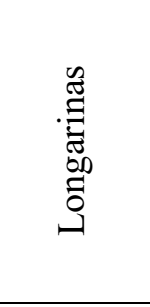 } & \multirow{6}{*}{3} & \multirow{3}{*}{ L1; L4 } & Corrosão de armaduras & 5 & 2 & 10 & \multirow{3}{*}{32,43} \\
\hline & & & Infiltração de água & 3 & 1 & 3 & \\
\hline & & & Manchas & 3 & 3 & 24 & \\
\hline & & \multirow{3}{*}{ L2; L3 } & Corrosão de armaduras & 5 & 2 & 10 & \multirow{3}{*}{14,74} \\
\hline & & & Infiltração de água & 3 & 1 & 3 & \\
\hline & & & Manchas & 3 & 2 & 6 & \\
\hline \multirow{10}{*}{$\frac{\mathscr{U}}{\tilde{a}}$} & \multirow{10}{*}{5} & \multirow{4}{*}{ P3; P4; P6; P7 } & Cobrimento deficiente & 3 & 2 & 6 & \multirow{4}{*}{35,48} \\
\hline & & & Corrosão de armaduras & 5 & 2 & 10 & \\
\hline & & & Desplacamento & 3 & 3 & 24 & \\
\hline & & & Manchas & 3 & 2 & 6 & \\
\hline & & \multirow{2}{*}{$\begin{array}{c}\text { P1; P2; P8; } \\
\text { P9; P10 }\end{array}$} & Desplacamento & 3 & 2 & 6 & \multirow{2}{*}{9,00} \\
\hline & & & Manchas & 3 & 2 & 6 & \\
\hline & & \multirow{4}{*}{ P5 } & Cobrimento deficiente & 3 & 2 & 6 & \multirow{4}{*}{76,10} \\
\hline & & & Corrosão de armaduras & 5 & 2 & 10 & \\
\hline & & & Desplacamento & 3 & 4 & 60 & \\
\hline & & & Manchas & 3 & 2 & 6 & \\
\hline \multirow{5}{*}{ 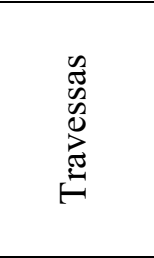 } & \multirow{5}{*}{5} & \multirow{2}{*}{ TV1; TV5 } & Infiltração de água & 3 & 1 & 3 & \multirow{2}{*}{8,00} \\
\hline & & & Manchas & 3 & 2 & 6 & \\
\hline & & \multirow{3}{*}{$\begin{array}{l}\text { TV2; TV3; } \\
\text { TV4 }\end{array}$} & Corrosão de armaduras & 5 & 2 & 10 & \multirow{3}{*}{14,74} \\
\hline & & & Infiltração de água & 3 & 1 & 3 & \\
\hline & & & Manchas & 3 & 2 & 6 & \\
\hline
\end{tabular}


Tabela 4 (continuação). Aplicação da metodologia GDE/UnB no viaduto do Itararé.

\begin{tabular}{|c|c|c|c|c|c|c|c|}
\hline Família & $\mathbf{F}_{\mathbf{r}}$ & Elementos & Manifestação Patológica & $\mathbf{F}_{\mathbf{p}}$ & $\mathbf{F}_{\mathbf{i}}$ & D & $\mathbf{G}_{\mathbf{d e}}$ \\
\hline \multirow{14}{*}{ Lajes } & \multirow{14}{*}{4} & \multirow{7}{*}{$\begin{array}{l}\text { VÃO 1; } \\
\text { VÃO } 4\end{array}$} & Cobrimento deficiente & 3 & 1 & 3 & \multirow{7}{*}{42,90} \\
\hline & & & Corrosão de armaduras & 5 & 2 & 10 & \\
\hline & & & Desagregação & 3 & 3 & 24 & \\
\hline & & & Desplacamento & 3 & 3 & 24 & \\
\hline & & & Fissuras & 2 & 2 & 4 & \\
\hline & & & Infiltração de água & 3 & 3 & 24 & \\
\hline & & & Manchas & 3 & 3 & 24 & \\
\hline & & \multirow{7}{*}{$\begin{array}{l}\text { VÃO 2; } \\
\text { VÃO } 3\end{array}$} & Cobrimento deficiente & 3 & 1 & 3 & \multirow{7}{*}{95,84} \\
\hline & & & Corrosão de armaduras & 5 & 2 & 10 & \\
\hline & & & Desagregação & 3 & 3 & 24 & \\
\hline & & & Desplacamento & 3 & 3 & 24 & \\
\hline & & & Fissuras & 2 & 2 & 4 & \\
\hline & & & Infiltração de água & 3 & 3 & 24 & \\
\hline & & & Manchas & 3 & 4 & 60 & \\
\hline \multirow{4}{*}{$\begin{array}{c}\text { Guarda- } \\
\text { corpo }\end{array}$} & \multirow{4}{*}{1} & \multirow{4}{*}{-} & Cobrimento deficiente & 3 & 3 & 24 & \multirow{4}{*}{147,37} \\
\hline & & & Corrosão de armaduras & 5 & 4 & 100 & \\
\hline & & & Desplacamento & 3 & 4 & 60 & \\
\hline & & & Manchas & 3 & 2 & 6 & \\
\hline \multirow{2}{*}{$\begin{array}{c}\text { Guarda- } \\
\text { rodas }\end{array}$} & \multirow{2}{*}{1} & \multirow{2}{*}{-} & Desagregação & 3 & 2 & 6 & \multirow{2}{*}{9,00} \\
\hline & & & Manchas & 3 & 2 & 6 & \\
\hline \multirow{3}{*}{$\begin{array}{l}\text { Pista de } \\
\text { rolamento }\end{array}$} & \multirow{3}{*}{1} & \multirow{3}{*}{-} & Descontinuidade & 5 & 3 & 40 & \multirow{3}{*}{66,67} \\
\hline & & & Desgaste superficial & 5 & 3 & 40 & \\
\hline & & & Desgaste da sinalização & 5 & 3 & 40 & \\
\hline \multirow{2}{*}{$\begin{array}{l}\text { Juntas de } \\
\text { dilatação }\end{array}$} & \multirow[b]{2}{*}{3} & \multirow{2}{*}{$\begin{array}{c}\mathrm{J} 1 ; \mathrm{J} 2 ; \mathrm{J} 3 ; \\
\mathrm{J} 4 ; \mathrm{J} 5\end{array}$} & Obstrução de junta & 5 & 3 & 40 & \multirow[b]{2}{*}{48,00} \\
\hline & & & $\begin{array}{l}\text { Desgaste do material de } \\
\text { preenchimento da junta }\end{array}$ & 5 & 2 & 10 & \\
\hline \multirow{2}{*}{\multicolumn{3}{|c|}{$\begin{array}{c}\text { GRAU DE } \\
\text { DETERIORAÇÃO DA } \\
\text { ESTRUTURA }\end{array}$}} & \multicolumn{5}{|c|}{$G_{d}=\frac{\sum_{i=1}^{k} K_{i}}{\sum_{i=1}^{k} F_{r, i}}=80,54$} \\
\hline & & & \multicolumn{5}{|c|}{$G_{d, \bmod }{ }^{*}=\frac{K_{m a ́ x}}{7,07} \cdot \sqrt{1+\frac{\left(\sum_{i=1}^{k} K_{i}\right)-K_{m a ́ x}}{\sum_{i=1}^{k} K_{i}}}=120,34$} \\
\hline
\end{tabular}

Foram utilizadas duas equações distintas para o cálculo do grau de deterioração da estrutura do viaduto. Como se observa na Tabela 4, o Grau de Deterioração encontrado a partir da primeira equação, de Fonseca (2007), foi de 80,54, indicando um nível sofrível que condiz mais com a realidade da estrutura do que o resultado obtido com a segunda equação, de Verly (2015), de 120,34. O nível sofrível, resultado encontrado com a equação de Fonseca (2007), indica a necessidade de planejamento de uma inspeção detalhada rigorosa visando uma intervenção de curto prazo (em 6 meses) na estrutura analisada neste estudo. 


\section{CONCLUSÕES}

A aplicação da metodologia GDE/UnB permitiu a obtenção de resultados satisfatórios e condizentes com a avaliação empírica da estrutura realizada durante a vistoria in loco, em que foram identificadas as manifestações patológicas presentes nos elementos estruturais. Além disso, foi possível identificar o nível das lesões e recomendar ações e intervenções adequadas ao nível de deterioração da estrutura. Os resultados obtidos indicaram um nível sofrível de degradação que denota a necessidade de planejamento de uma inspeção detalhada rigorosa visando uma intervenção de curto prazo (em até 6 meses) na estrutura analisada. Essas medidas possibilitariam atenuar as principais patologias identificadas que se desenvolveram principalmente pela falta de manutenção periódica, resultante da ausência de um sistema de gerenciamento de OAE como esta que possui importância significativa na estrutura viária da cidade, uma vez que faz a ligação entre dois bairros principais. Destaca-se, portanto, a viabilidade da aplicação da metodologia GDE/UnB no levantamento de dados capazes de definir a situação atual desta e de outras estruturas, resultando em um planejamento de ações de correção compatível com cada caso.

\section{REFERÊNCIAS}

Castro, E. K. (1994), "Desenvolvimento de metodologia para manutenção de estruturas de concreto armado", Dissertação (Mestrado), Departamento de Engenharia Civil e Ambiental, Universidade de Brasília, Brasília, 185 p.

Euqueres, P. (2011), “Metodologia de inspeção de pontes de concreto armado”, Dissertação (Mestrado), Escola de Engenharia Civil, Universidade Federal de Goiás, Goiânia, 168 p.

Fonseca, R. P. (2007), "A estrutura do Instituto Central de Ciências: aspectos históricos, científicos e tecnológicos de projeto, execução, intervenções e propostas de manutenção”, Dissertação (Mestrado), Departamento de Engenharia Civil e Ambiental, Universidade de Brasília, Brasília, 213 p.

Lopes, B. A. R. (1998), "Sistema de manutenção predial para grades estoques de edifícios: estudo para inclusão do componente "Estrutura de Concreto"". Dissertação (Mestrado), Departamento de Engenharia Civil e Ambiental, Universidade de Brasília, Brasília, 308 p.

Verly, R. C. (2015), "Avaliação de metodologias de inspeção como instrumento de priorização de intervenções em obras de arte especiais", Dissertação (Mestrado), Departamento de Engenharia Civil e Ambiental, Universidade de Brasília, Brasília, 178 p. 